\title{
Einsatz der Cyclovoltammetrie zur Steigerung der Selektivität von Festelektrolytsensoren
}

\author{
Anastasiya Ruchets ${ }^{1}$, N. Donker ${ }^{2}$, D. Schönauer-Kamin'2, R. Moos², J. Zosel', U. Guth ${ }^{3}$, M. Mertigi,3 \\ ${ }^{1}$ Kurt-Schwabe-Institut für Mess- und Sensortechnik e. V. Meinsberg, Kurt-Schwabe-Straße 4, \\ 04736 Waldheim, Germany \\ ${ }^{2}$ Lehrstuhl für Funktionsmaterialien, Universität Bayreuth, 95440 Bayreuth, Germany \\ ${ }^{3}$ Professur für Physikalische Chemie, Mess- und Sensortechnik, Technische Universität Dresden, \\ 01062 Dresden, Deutschland
}

\section{Zusammenfassung}

Mit einem kommerziellen Festelektrolytsensor (FES) auf der Basis von stabilisiertem Zirconiumdioxid wurden unter Verwendung der Cyclovoltammetrie die redoxaktive Gase wie $\mathrm{H}_{2}, \mathrm{O}_{2}$ und $\mathrm{H}_{2} \mathrm{O}$, verdünnt in $\mathrm{N}_{2}$, bestimmt. Die erzielten Ergebnisse zeigen, dass parallel sowohl $\mathrm{H}_{2}$ als auch $\mathrm{O}_{2}$ quantitativ und mit hoher Selektivität bei verschiedenen $\mathrm{H}_{2} \mathrm{O}$-Konzentrationen mittels Cyclovoltammetrie gemessen werden können. Die wasserstoffabhängigen Peaks treten in den cyklischen Voltammogrammen (CV) in anodischer Scan-Richtung bei Potentialen zwischen 0,4 und 0,2 V gegen eine Pt/Luft-Referenzelektrode bei Scangeschwindigkeiten zwischen 10 und $100 \mathrm{mV} / \mathrm{s}$ im Temperaturbereich $700-750{ }^{\circ} \mathrm{C}$ auf. Die untere Nachweisgrenze für $\mathrm{H}_{2}$ liegt unter $5 \mathrm{Vol}$-ppm während die obere Nachweisgrenze etwa 100 Vol.-ppm beträgt. Dieser Messbereich wurde für $\mathrm{O}_{2}$-Konzentrationen 0 - 200 Vol.-ppm und $\mathrm{H}_{2} \mathrm{O}-$ Konzentrationen 0 - 600 Vol.-ppm ermittelt.

Keywords: Cyclovoltammetrie, Gassensoren, Wasserstoff, Yttriumoxid-stabilisiertes Zirconiumdioxid (YSZ), Pt-Elektroden.

\section{Einführung}

Festoxid-Elektrolyte wie Yttriumoxid-stabilisiertes Zirconiumdioxid (YSZ) mit hoher Sauerstoffionenleitfähigkeit und geringer elektronischer Leitfähigkeit sind ausgezeichnete Materialien für Festelektrolyt-Sauerstoffsensoren bei mittleren und hohen Temperaturen.

Solche Festelektrolytsensoren (FES) können im stationären oder dynamischen Modus betrieben werden. Je nachdem, ob es sich bei dem Ausgangssignal um eine Spannung oder einen elektrischen Strom handelt, können die im stationären Modus betriebenen elektrochemischen Gassensoren als potentiometrisch oder amperometrisch klassifiziert werden [1]. Da Nichtgleichgewichts-Gasgemische an den heißen und katalytisch hochaktiven Pt-Elektroden dieser Sensoren sofort equilibriert werden, sind diese Sensoren im stationären Modus nicht für einzelne Komponenten selektiv, was für manche Applikationen einen gravierenden Nachteil darstellt [2].

Ein dynamischer Modus umfasst alle Methoden, bei denen die Messelektrode zeitabhängig polarisiert und die Antwort des Sensors ebenfalls zeitabhängig gemessen wird. Beispiele für diesen Modus sind die Pulspolarisation [3], die Cyclovoltammetrie [4] oder die
Impedanzspektroskopie (EIS) [5]. Es wurde bereits gezeigt, dass bei dynamisch betriebenen Sensoren eine Verbesserung der Selektivität erreicht werden kann, weil unterschiedliche Kinetiken der Elektrodenreaktionen genutzt und so Einzelkomponenten unabhängig voneinander erfasst werden können [6]. In dieser Arbeit wird die Eignung der Cyclovoltammetrie beim Betrieb eines rohrförmigen FES für die Steigerung der Selektivität in Nichtgleichgewichts-Gasgemischen untersucht. Der FES ist mit zwei Pt-Elektroden ausgestattet, wobei die Arbeitselektrode auf der Rohrinnenseite in der Messgasatmosphäre und die Referenz- und Gegenelektrode auf der Rohraußenseite an Luft positioniert sind. Es konnte bereits gezeigt werden $[4,6]$, dass mit Hilfe der Cyclovoltammetrie an solchen FES eine selektive Messung in redoxaktiven Gasgemischen möglich wird. Der vorliegende Beitrag hat zum Ziel, die Messbereichsgrenzen für diese Methode genauer zu quantifizieren.

\section{Experimenteller Aufbau}

Der gelb hinterlegte Teil des in Abb. 1 gezeigten Versuchsaufbaus ist für die gleichzeitige und unabhängige Einstellung der Konzentrationen von $\mathrm{O}_{2}, \mathrm{H}_{2}$ und $\mathrm{H}_{2} \mathrm{O}$ in Stickstoff-Gasgemischen vorgesehen. 


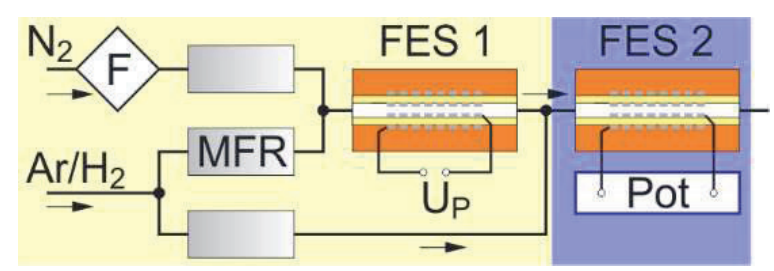

Abb 1: $\quad$ Schema des Versuchsaufbaus, FES = Festelektrolytsensor, Pot $=$ Potentiostat, MFR = Masseflussregler, $F=$ Filter für $\mathrm{O}_{2}, \mathrm{H}_{2} \mathrm{O}$ und Kohlenwasserstoffe.

$\mathrm{O}_{2}$ und $\mathrm{H}_{2} \mathrm{O}$ wurden durch Sauerstoffelektrolyse in den wasserstoffhaltigen Stickstoff im ersten Festelek-trolytsensor (FES 1) fixiert. Der blau hinterlegte zweite Festelektrolytsensor (FES 2, beide FES vom Typ O2-DF 28.0, ZIROX - Sensoren \& Elektronik $\mathrm{GmbH}$, Greifswald, Deutschland) wurde für dynamische elektrochemische Messungen verwendet. Beide Sensoren vom Typ "Messgas, Pt|YSZ|Pt, Luft" weisen zwei $4 \mathrm{~cm}$ lange zylindrische Elektroden auf, die aus einem reinem Pt-Netz (250 mesh) bestehen und mit poröser YSZ-Paste auf den Innen- und Außenflächen des YSZ-Rohres (8 mol-\% Yttriumoxid, dinnen $=3,9 \mathrm{~mm}$ (Arbeitselektrode) und daußen $=5,9 \mathrm{~mm}$ (Referenz-/Gegenelektrode) gesintert wurden. Alle Gasversorgungsleitungen sind mit Edelstahlrohren und Verschraubungen (Swagelok, Solon, OH, USA) ausgestattet. Die Anschlüsse beider FES an die Gasversorgung wurden so modifiziert, dass die He-Leckrate der FES von $10^{-6}$ auf $10^{-9}$ mbar $\cdot$ L/s verringert wurde.

Da die signalrelevante Kinetik in hohem Maß von der Elektrodenmorphologie beeinflusst wird, wurde diese mittels Rasterelektronenmikroskopie (REM) untersucht. Die in Abb. 2 aufgeführten REM-Aufnahmen belegen die Anbindung der Elektrodendrähte an den Festelektrolyten durch eine poröse YSZ-Schicht. Diese poröse Schicht bedeckt große Teile der Messund Referenzelektrode und bietet eine erweiterte Dreiphasengrenze (DPG), an welcher der Sauerstofftransfer zwischen Gas und Festelektrolyt erfolgt. Das Messgas diffundiert teilweise durch diese poröse Schicht, um die DPG zu erreichen.

Die Durchflussmengen von Stickstoff 5,0 und Wasserstoff-Testgas (0,1 Vol.- $\% \mathrm{H}_{2}$ in $\left.\mathrm{Ar}\right)$ wurden mit Masseflussreglern (Brooks Instrument Company, Hatfield, USA) eingestellt. Das Verdünnungsgas Stickstoff wurde durch einen Dreifachfilter für Wasserdampf, Sauerstoff und Kohlenwasserstoffe (Restek $\mathrm{GmbH}$, Bad Homburg) geleitet, wodurch dessen Reinheit auf einen Grad > 6,0 angehoben wird.

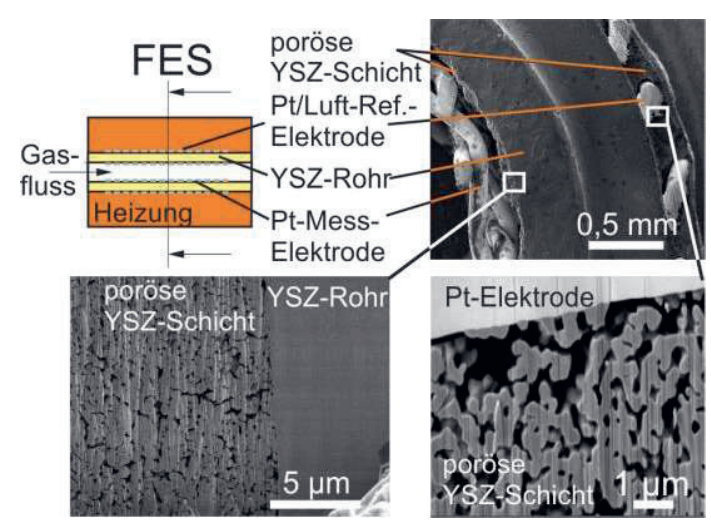

Abb 2: Schema und REM-Aufnahmen von Schnitten durch ein Festelektrolytrohr (8 mol-\% YSZ) eines FES mit beidseitig aufgesinterten Pt-Elektroden in einer porösen YSZ-Schicht.

Die elektrochemischen Messungen an FES 2 wurden mit einem elektrochemischen Mehrzweck-Messsystem Reference 600 ${ }^{\mathrm{TM}}$ (Gamry Instruments, Warminster, USA) durchgeführt.

\section{Ergebnisse und Diskussion}

\section{Einfluss der Scangeschwindigkeit}

Die in Abb. 3 dargestellten CV wurden in einem nicht-equilibrierten Gasgemisch mit $\varphi\left(\mathrm{H}_{2}\right)=50$ Vol.-ppm, $\varphi\left(\mathrm{O}_{2}\right)=10$ Vol.-ppm und $\varphi\left(\mathrm{H}_{2} \mathrm{O}\right)<10$ Vol.-ppm bei unterschiedlichen Scangeschwindigkeiten und der FES-Temperatur $750{ }^{\circ} \mathrm{C}$ gemessen. Sie bestätigen die in [4] dargestellten Ergebnisse, die ebenfalls belegen, dass wasserstoffbedingte Peaks in anodischer Scanrichtung zwischen $-0,4$ und $-0,2 \mathrm{~V}$ bei Scangeschwindigkeiten zwischen 10 und $100 \mathrm{mV} / \mathrm{s}$ auftreten.

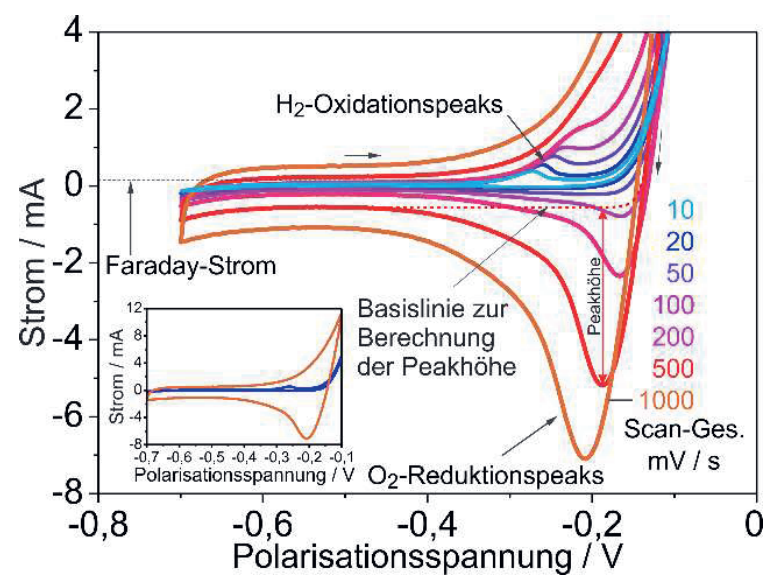

Abb 3: $\quad C V$ verschiedener Scangeschwindigkeiten; Durchfluss $=10 \mathrm{ml} / \mathrm{min}$, $\varphi\left(H_{2}\right)=50 \mathrm{Vol}$. $-p p m, \varphi\left(\mathrm{O}_{2}\right)=10 \mathrm{Vol}$.ppm, $\varphi\left(\mathrm{H}_{2} \mathrm{O}\right)<1$ Vol.-ppm, Sensortemperatur $=750^{\circ} \mathrm{C}$, Inset-Diagramm: CV bei 20 und $1000 \mathrm{mV} / \mathrm{s}$ im gesamten Strommessbereich. 
Im Gegensatz dazu entwickeln sich die sauerstoffbedingten Peaks bei Scangeschwindigkeiten über $100 \mathrm{mV} / \mathrm{s}$ in kathodischer Scanrichtung zwischen $-0,1$ und $-0,45 \mathrm{~V}$.

Diese Unterschiede zwischen beiden Reaktionskinetiken ermöglichen einen unabhängigen und selektiven Nachweis beider Gase im untersuchten Konzentrationsbereich.

In Anbetracht der Tatsache, dass die katalytisch unterstützte Wasserstoffoxidation an heißen Pt-Oberflächen mit sehr hohen Geschwindigkeiten abläuft, war es überraschend, bei diesen vergleichsweise niedrigen Scangeschwindigkeiten einen ausgeprägten wasserstoffbedingten Oxidationspeak zu finden. Aus diesem Ergebnis lässt sich vermuten, dass dieser Analyt in der Nähe der Elektrode während der kathodischen Polarisation gespeichert und aus diesem Speicher beim Rücklauf in anodische Richtung verzögert freigegeben wird.

Um die verschiedenen gemessenen Peaks mit den Versuchsbedingungen zu korrelieren, wurden die entsprechenden Peakhöhen berechnet, indem der Strom einer Basislinie vom Peakstromwert abgezogen wurde. Dies ist in Abb. 3 für den Sauerstoffpeak bei 10 - 1000 $\mathrm{mV} / \mathrm{s}$ dargestellt. Die Basislinien wurden berechnet, indem eine Exponentialfunktion an die kathodische CV-Kurve in den Potentialbereichen $(-0,1--0,15 \mathrm{~V})$ und $(-0,5--0,55 \mathrm{~V})$ für die $\mathrm{O}_{2}$-Peaks sowie in den anodischen Bereichen $(-0,5--0,45 \mathrm{~V})$ und $(-0,15--0,1 \mathrm{~V})$ für die $\mathrm{H}_{2}$-Peaks angepasst wurde.

Um mehr über die kinetischen Limitierungen der Elektrodenreaktionen zu erfahren, wurde die Abhängigkeit der Peakhöhe von der Scangeschwindigkeit untersucht.

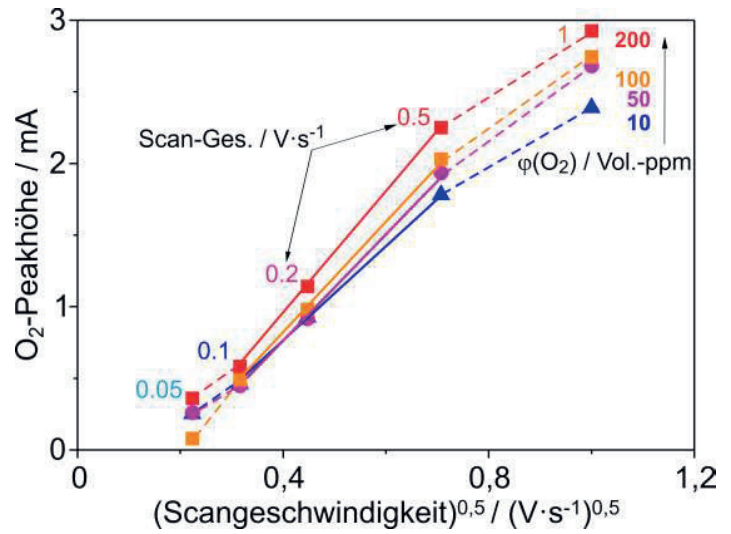

Abb. 4: Abhängigkeit der $\mathrm{O}_{2}$-Peakhöhen von der Quadratwurzel der Scangeschwindigkeit; $\varphi\left(\mathrm{H}_{2}\right)=50 \mathrm{Vol}$.-ppm, $\varphi\left(\mathrm{O}_{2}\right)=10,50,100,200$ Vol.-ppm, $\varphi\left(\mathrm{H}_{2} \mathrm{O}\right)<1$ Vol.-ppm, Sensortemperatur $=650^{\circ} \mathrm{C}$, Vol.str. $=10 \mathrm{ml} / \mathrm{min}$.
Wenn die Peakhöhe linear mit der Quadratwurzel der Scangeschwindigkeit zunimmt, wie es die Randles-Ševčik-Gleichung (RSG) [7, 8] beschreibt, liegt eine kinetische Limitierung der elektrochemischen Reaktion durch Diffusion vor. Diese Abhängigkeit wurde bereits für die $\mathrm{H}_{2}$-bezogenen Peaks gefunden [4].

In Abb. 4 ist dargestellt, dass die RSG diese Abhängigkeit auch für die $\mathrm{O}_{2}$-bezogenen Peaks bei Scangeschwindigkeiten zwischen 100 und $500 \mathrm{mV} / \mathrm{s}$ beschreibt. Außerhalb dieses Bereichs wirken andere Prozesse wie der Ladungstransfer begrenzend und führen so zu einer Abweichung vom durch die RSG beschriebenen linearen Verlauf.

Mit der RSG ist eine Abschätzung des $\mathrm{O}_{2}$ Diffusionskoeffizienten aus dem Geradenanstieg möglich. Die größte Unsicherheit dieser Schätzung betrifft die Elektrodenfläche $A$, die aus den REM-Aufnahmen der in eine poröse YSZ-Schicht eingebetteten Elektrode auf $A=3,5 \mathrm{~cm}^{2}$ geschätzt wurde. Der resultierende Diffusionskoeffizient $D=2,6 \mathrm{~cm}^{2} / \mathrm{s}$ ist etwas höher als der $\mathrm{O}_{2}$-Diffusionskoeffizient in der an die Elektrode angrenzenden Gasphase, der sich aus der Chapman-Enskog-Gleichung [9] zu $\mathrm{D}\left(\mathrm{O}_{2}\right.$ in $\left.\mathrm{N}_{2}\right)=1,4 \mathrm{~cm}^{2} / \mathrm{s}$ bei $650{ }^{\circ} \mathrm{C}$ und 1 bar berechnen lässt. Die $\mathrm{O}_{2}$-Umsatzlimitierung erfolgt demnach vor allem durch Gasdiffusion an der Arbeitselektrode und weniger durch Oberflächendiffusion.

Durch Änderung der Scangeschwindigkeit lassen sich somit selektiv und nahezu parallel Wasserstoff und Sauerstoff im Gasgemisch nachweisen. Im Folgenden werden die Bereichsgrenzen dieser Methode beschrieben.

\section{Einfluss von Volumenstrom und Tempera- tur}

In Abb. 5 ist der Einfluss des Volumenstroms auf die $\mathrm{CV}$ bei drei FES-Temperaturen sowie $\varphi\left(\mathrm{H}_{2}\right)=\varphi\left(\mathrm{O}_{2}\right)=50$ Vol.-ppm dargestellt. Mit zunehmendem Volumenstrom steigt die Menge an überschüssigem Sauerstoff, der die Elektrode pro Zeiteinheit erreicht. Dies führt zu einer Verschiebung der CV zu negativeren Stromwerten. Bei Temperaturen zwischen 700 und $750{ }^{\circ} \mathrm{C}$ wird am kathodischen Wendepunkt ein zunehmender Reduktionsstrom beobachtet, der bei geringen Volumenströmen am deutlichsten ausgeprägt ist. Dies deutet darauf hin, dass freier Sauerstoff den $\mathrm{H}_{2}$-Speicherprozess beeinflusst.

Mit diesem Anstieg des Reduktionsstroms bei abnehmendem Volumenstrom und steigender Temperatur steigt auch die Höhe der $\mathrm{H}_{2}$ Oxidationspeaks. 


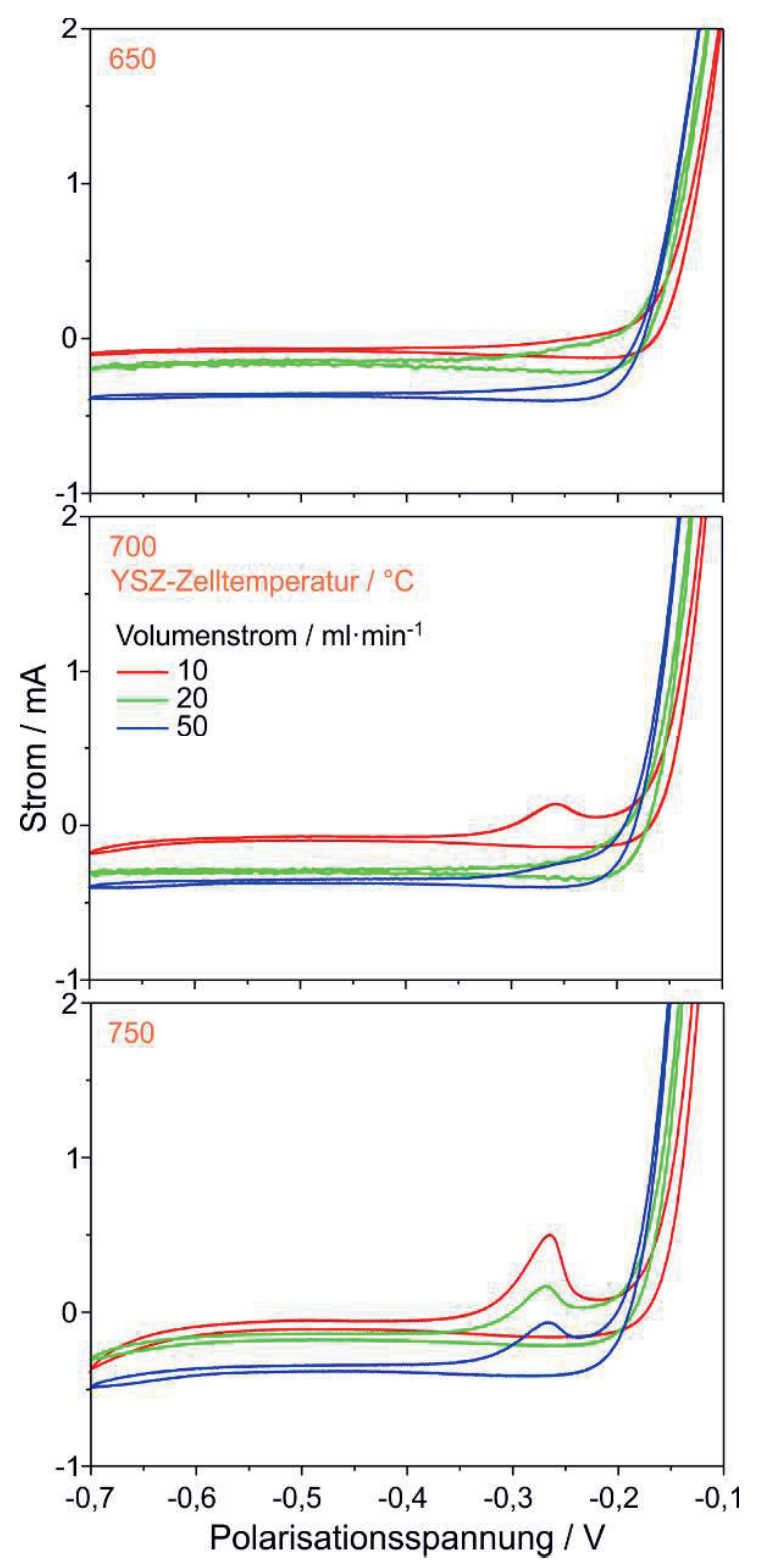

Abb. 5: CV bei verschiedenen Volumenströmen und Sensortemperaturen; Scangeschwindigkeit $=20 \mathrm{mV} / \mathrm{s}, \varphi\left(\mathrm{O}_{2}\right)=$ 50 Vol.-ppm, $\varphi\left(H_{2}\right)=50$ Vol. $-p p m$, $\varphi\left(\mathrm{H}_{2} \mathrm{O}\right)<1 \mathrm{Vol}$.-ppm.

\section{Wiederholgenauigkeit der Methode}

Um die Wiederholgenauigkeit des $\mathrm{H}_{2}$-Peaks zu untersuchen, wurden 30 identische CV mit einer Minute Pause zwischen zwei aufeinanderfolgenden CV durchgeführt. Aus dieser Messung sind Beispiele in Abb. 6 dargestellt. Diese zeigen, dass der Wasserstoffpeak mit zunehmender Zyklenzahl abnimmt, bis nach etwa 20-25 Zyklen ein konstanter Wert erreicht wird. Weiterhin wurde gefunden, dass sich diese Änderungen zwischen aufeinanderfolgenden $\mathrm{CV}$ dadurch verringern lassen, dass der Sensor zwischen zwei CV kurzzeitig mit der vor dem ersten CV gemessenen Gleichgewichts-Spannung $\cup_{\text {eq }}$ polarisiert wird.

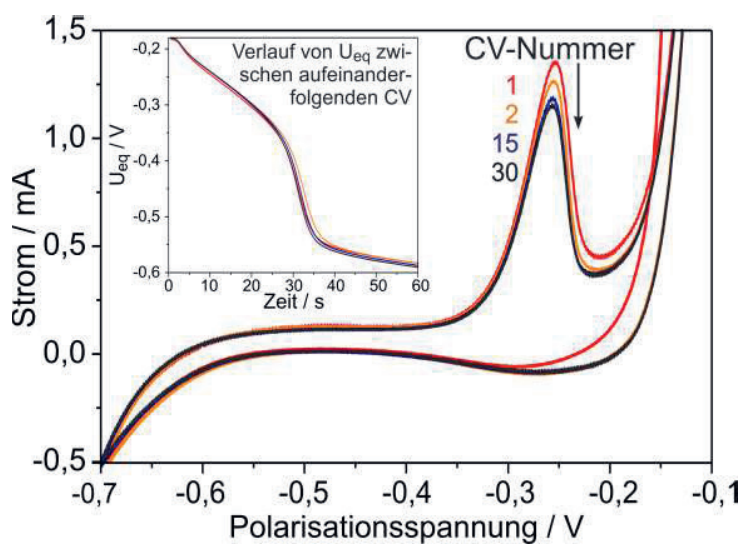

Abb. 6: Beispiele von $30 \mathrm{CV}$ bei konstanten Bedingungen mit einer Minute Pause zwischen zwei aufeinanderfolgenden $\mathrm{CV}$; Sensortemperatur $=750^{\circ} \mathrm{C}$, Volumenstrom $=10 \mathrm{ml} / \mathrm{min}$, Scangeschwindigkeit $=20 \mathrm{mV} / \mathrm{s}, \varphi\left(\mathrm{O}_{2}\right)=$ 0,2 Vol.-ppm, $\varphi\left(H_{2}\right)=50$ Vol.-ppm, $\varphi\left(\mathrm{H}_{2} \mathrm{O}\right)<1$ Vol.-ppm, Inset-Diagramm: zwischen zwei CV gemessene Gleichgewichtsspannung $U_{\text {eq. }}$.

Um die Reproduzierbarkeit der Methode zu verbessern, wurden automatisierte Abläufe programmiert und immer die CV mit der gleichen Zyklusnummer verglichen.

\section{Randbedingungen für die Wasserstoffde- tektion}

\section{Messbereich der $\mathrm{H}_{2}$-Konzentration}

Ausgewählte $\mathrm{CV}$ in Messgasen unterschiedlicher $\mathrm{H}_{2}$-Konzentration mit $\varphi\left(\mathrm{O}_{2}\right)=0,2$ Vol.ppm sind in Abb. 7 dargestellt. Die Peakauswertung erfolgte wie oben für Sauerstoffpeaks beschrieben durch Subtraktion der exponentiell ansteigenden Basislinie.

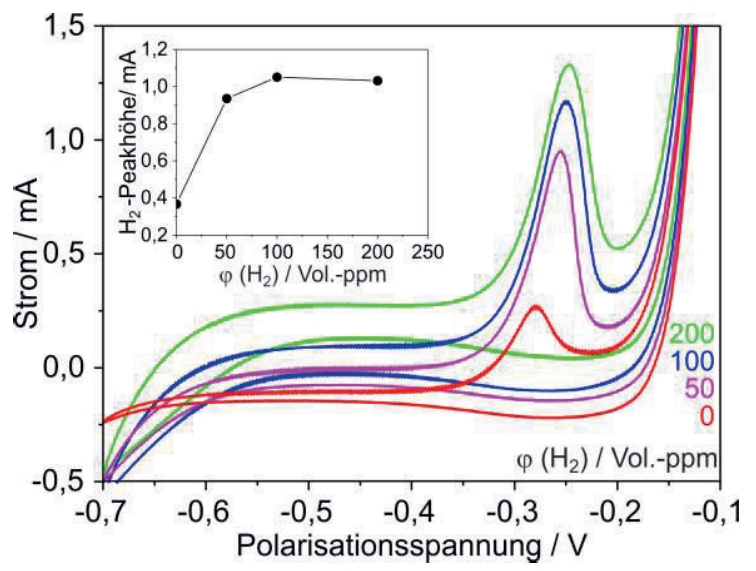

Abb 7: $\quad \mathrm{CV}$ bei verschiedenen $\mathrm{H}_{2}$-Konzentrationen; Sensortemp. $=750^{\circ} \mathrm{C}$, Volumenstrom $=10 \mathrm{ml} / \mathrm{min}$, Scangeschwindigkeit $=20 \mathrm{mV} / \mathrm{s}, \varphi\left(\mathrm{O}_{2}\right)=$ 0,2 Vol.-ppm, Inset-Diagramm: $\mathrm{H}_{2^{-}}$ bezogene Peakhöhe. 
Die im Inset von Abb. 7 dargestellten resultierenden Peakhöhen steigen bis $\mathrm{zu}$ einer $\mathrm{H}_{2}-$ Konzentration von etwa 100 Vol.-ppm an und verlaufen danach nahezu konstant. Weiterhin verschieben sich die $\mathrm{CV}$ mit zunehmender $\mathrm{H}_{2-}$ Konzentration in anodische Stromrichtung, da im gesamten Scanbereich ein potentialunabhängiger Faradayscher Stromfluss überlagert ist. Ähnliche Experimente zur Charakterisierung der $\mathrm{O}_{2}$-Querempfindlichkeit der $\mathrm{H}_{2}-$ Peaks sind in [6] für den Temperaturbereich $700-750^{\circ} \mathrm{C}$ bei $\varphi\left(\mathrm{O}_{2}\right)=0,2-200$ Vol.-ppm beschrieben.

\section{Einfluss der $\mathrm{H}_{2} \mathrm{O}$-Konzentration}

In Abb. 8 sind Ergebnisse zum Einfluss der $\mathrm{H}_{2} \mathrm{O}$-Konzentration auf die $\mathrm{H}_{2}$-Detektion bei niedrigen Scangeschwindigkeiten dargestellt. Im untersuchten $\mathrm{H}_{2} \mathrm{O}$-Konzentrationsbereich zwischen 0 - 600 Vol.-ppm wurden zunächst ansteigende $\mathrm{H}_{2}$-Peakhöhen gefunden, die später mit weiter zunehmender $\mathrm{H}_{2} \mathrm{O}$-Konzentration wieder abnehmen. Der bei kleinen $\mathrm{H}_{2} \mathrm{O}-$ Konzentrationen $<400$ Vol.-ppm gefundene Anstieg der $\mathrm{H}_{2}$-Peakhöhe könnte auf eine Intensivierung der Oberflächendiffusion von $\mathrm{H}_{2}$ in den Zwischenspeicher zurückzuführen sein. Weiterhin tritt bei steigender $\mathrm{H}_{2} \mathrm{O}$-Konzentration eine leichte Peak-Verbreiterung auf und die Stromwerte im Potentialbereich zwischen 0,4 und $-0,7 \mathrm{~V}$ werden nach unten verschoben. Die bei $\mathrm{H}_{2} \mathrm{O}$-Konzentrationen > 400 Vol.-ppm beobachtete Abnahme der $\mathrm{H}_{2}$-Peakhöhe wird dagegen wahrscheinlich von anderen Vorgängen verursacht, z.B. könnte eine erhöhte Konkurrenz-Adsorption von Wassermolekülen an der Elektrode den $\mathrm{H}_{2}$-Übertritt hemmen.

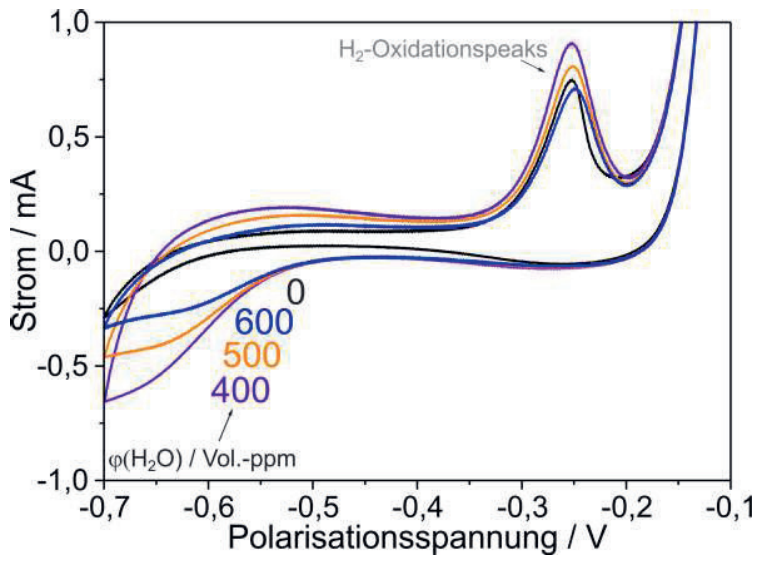

Abb 8: $\quad C V$ bei verschiedenen $\mathrm{H}_{2} \mathrm{O}-\mathrm{Kon}$ zentrationen; $\varphi\left(\mathrm{H}_{2}\right)=50$ Vol.- $\mathrm{ppm}$, $\varphi\left(\mathrm{O}_{2}\right)=10-15 \mathrm{Vol}$.-ppm, Sensortemperatur $=750{ }^{\circ} \mathrm{C}$, Scangeschwindigkeit $=20 \mathrm{mV} / \mathrm{s}$, Volumenstrom = $10 \mathrm{ml} / \mathrm{min}$
In [10] wird beschrieben, wie die Oberfläche einer Pt-Mikroelektrode mit $\mathrm{H}_{2} \mathrm{O}$-Molekülen bedeckt ist, wenn die $\mathrm{H}_{2} \mathrm{O}$-Konzentration im Gas ansteigt. Darüber hinaus beeinflusst Wasser auch die Wasserstofflöslichkeit im Festelektrolyt [11, 12]. Wasser wandert auch selbst in den Elektrolyt ein [13].

Dennoch lässt sich der $\mathrm{H}_{2}$-bezogene Peak auch bei relativ hohen $\mathrm{H}_{2} \mathrm{O}$-Konzentrationen mit hoher Sensitivität nachweisen. Der Anstieg des Reduktionsstroms im Potentialbereich zwischen -0,6 und -0,7 V eröffnet die Möglichkeit, neben $\mathrm{H}_{2}$ und $\mathrm{O}_{2}$ auch $\mathrm{H}_{2} \mathrm{O}$ bei höheren Konzentrationen in den Gasgemischen messtechnisch zu erfassen.

\section{Einfluss der $\mathrm{O}_{2}$-Konzentration}

Der in [6] beschriebene Einfluss der Sauerstoffkonzentration im Bereich $\varphi\left(\mathrm{O}_{2}\right)=0-200$ Vol.-ppm auf die Peakhöhe der gemessenen $\mathrm{H}_{2}$-Peaks ist auch bei hohen Sauerstoffüberschüssen so gering, dass die $\mathrm{H}_{2}$-Peaks sicher nachweisbar bleiben. Steigt die $\mathrm{O}_{2}$-Konzentration über diesen Bereich hinaus an, sollten sich die $\mathrm{H}_{2}$-Peaks verringern und schließlich verschwinden, da die $\mathrm{H}_{2}$-Oxidation in der Gasphase immer mehr beschleunigt wird. Diese Vermutung wird durch die in Abb. 9 gezeigten CV bestätigt. Im Bereich $\varphi\left(\mathrm{O}_{2}\right)=500-1000$ Vol.-ppm sind keine $\mathrm{H}_{2}$-Peaks mehr nachweisbar.

Möglicherweise lassen sich auch bei höheren Sauerstoffkonzentrationen als 200 Vol.-ppm noch Wasserstoffpeaks finden, wenn der negative Umkehrpunkt der CV zu noch negativeren Potentialen verschoben wird. Diese Untersuchungen sind geplant.

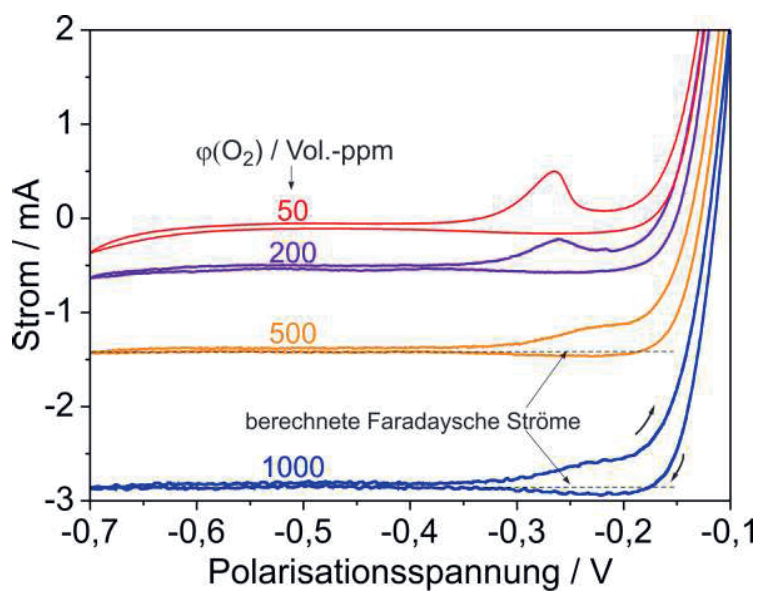

Abb 9: $\quad \mathrm{CV}$ bei erhöhten $\mathrm{O}_{2}$-Konzentrationen; $\varphi\left(\mathrm{H}_{2}\right)=50 \mathrm{Vol}$.-ppm, $\varphi\left(\mathrm{H}_{2} \mathrm{O}\right)$ $<1 \mathrm{Vol} .-\mathrm{ppm}$, Sensortemperatur = $750^{\circ} \mathrm{C}$, Scangeschwindigkeit $=20$ $\mathrm{mV} / \mathrm{s}$, Volumenstrom $=10 \mathrm{ml} / \mathrm{min}$. 


\section{Schlussfolgerungen}

Die Cyclovoltammetrie als dynamische Polarisationsmethode wurde an einem kommerziell verfügbaren Festelektrolytsensor angewendet, der im Durchflussmodus betrieben wurde. Durch den Einsatz der Cyclovoltammetrie und die Verwendung von Gasgemischen mit unterschiedlichen Wasserstoff-, Sauerstoff- und Wasserdampfkonzentrationen wurde nachgewiesen, dass es möglich ist, zwischen den untersuchten Redoxkomponenten mit dieser dynamischen Methode in nicht-equilibrierten Gemischen durch Variation experimenteller Parameter wie Scangeschwindigkeit und Sensortemperatur zu unterscheiden. Eine unabhängige quantitative Bestimmung kleiner $\mathrm{H}_{2}$ und $\mathrm{O}_{2}$-Konzentrationen ist auch bei höheren Überkonzentrationen des jeweiligen Reaktionspartners mit hoher Selektivität und Sensitivität möglich. In dieser Arbeit wurden die Messbereichsgrenzen einer solchen selektiven Messung näher bestimmt. Dabei ließ sich zeigen, dass der $\mathrm{H}_{2}$-Nachweis bis $\mathrm{zu} 100$ Vol.ppm bei Volumenströmen zwischen 10 und $20 \mathrm{ml} / \mathrm{min}$ und $\mathrm{O}_{2}$-Konzentrationen im Bereich von 0 bis 200 Vol.-ppm sowie in Gegenwart von Wasserdampf mit $0-600$ Vol.-ppm im untersuchten Potentialbereich zwischen -0,7 und $-0,1 \mathrm{~V}$ möglich ist.

\section{Danksagung}

Die Arbeit wird von der Deutschen Forschungsgemeinschaft (DFG) im Rahmen des Projekts "Dynamische Methoden für elektrochemische Gassensoren (DynaSens)" (MO 1060/30-1, ZO 139/3-1) gefördert.

Die Autoren danken J. Posseckardt vom KSI für die Anfertigung der REM-Aufnahmen. Darüber hinaus danken die Autoren Herrn F. Altmann und seinem Team am Fraunhofer-Institut für Mikrostrukturierung von Materialien und Systemen (IMWS), Halle, für die Durchführung von materialbezogenen Untersuchungen und Analysen.

\section{Literatur}

[1] S. Zhuiykov, Electrochemistry of Zirconia Gas Sensors. CRC Press, Boca Raton (2007)

[2] H.-H. Möbius, Solid-State Electrochemical Potentiometric Sensors for Gas Analysis, in: W. Göpel et al. (Eds.), Sensors A comprehensive survey, Vol. 3, VCH, Weinheim (1991)

[3] S. Fischer, R. Pohle, E. Magori, M. Fleischer, R. Moos, Solid State lonics 262, 288-291 (2014); doi: 10.1016/j.ssi.2014.01.022

[4] M. Schelter, J. Zosel, W. Oelßner, U. Guth, M. Mertig, Journal of Sensors and Sensor Systems 5, 319-324 (2016); doi: 10.5194/jsss-5-3192016

[5] H. Pöpke, E. Mutoro, C. Raiß, B. Luerßen, M. Amati, M.K. Abyaneh, L. Gregoratti, J. Janek, Electrochim. Acta 56, 10668-10675 (2011); doi: 10.1016/j.electacta.2011.04.057.

[6] A. Ruchets, N. Donker, D. Schönauer-Kamin, R. Moos, J. Zosel, U. Guth, M. Mertig Sensors \& Actuators: B. Chemical 290, 53-58 (2019); doi: 10.1016/j.snb.2019.03.063

[7] J. E. B. Randles, Trans. Faraday Soc., 44, 327338 (1948); doi: 10.1039/TF9484400327

[8] A. Sevcik, Collect. Czech. Chem. Commun. 13, 349-377 (1948); doi: 10.1135/cccc19480349

[9] E. I. Cussler, Cambridge University Press, Cambridge 104-108 (1997); ISBN 052156477

[10] C. Vonau, Dissertation, Technische Universität Dresden, 151 (2015); http://nbnresolving.de/urn:nbn:de:bsz:14-qucosa-183965.

[11] K. Park, D.R. Olander, J. Am. Ceram. Soc. 74 (1) (1991); 72-77, doi: 10. 1111/j.11512916.1991.tb07299.x.

[12] S. Yamanaka, T. Nishizaki, M. Uno, M. Katsura, J. Alloys Compd. 293-295, 38-41 (1999); doi: 10.1016/S0925-8388(99)00396-5.

[13] C. Wagner, Berichte der Bunsengesellschaft für physikalische Chemie 72 (7), 778-781 (1968); doi: 10.1002/bbpc.19680720709. 